\title{
DESIGN OF A LASER SOURCE USING A TRAVELLING WAVE OPTICAL AMPLIFIER WITH EXTERNAL FEEDBACK
}

\author{
S.Ruiz-Moreno, M.A.Acero, M.J.Soneira \& J.Guitart \\ Signal Theory and Communications Dep., E.T.S.I.T.,Barcelona (UPC) \\ $\mathrm{C} / J o r g e$ Girona Salgado $\mathrm{s} / \mathrm{n}, 08034$ Barcelona, Spain
}

\begin{abstract}
The main aim of this paper is to analyse the performance of an optical source designed starting from the external feedback of a travelling wave optical amplifier (TWOA). The proposed structure is analised using a computer model based on travelling wave and carrier equations. From the performed simulations characteristical curves (output power, spectral quality, etc) have been obtained, which show the high performances that this type of source can of fer in optical fibre communication systems. On the other hand, we can infer interesting conclusions from the obtained results. This will enable us to achieve optimal designs regarding maximum optical power and spectral quality values.
\end{abstract}

\section{Introduction}

The great advance that the use of optical fibres has meant in long haul transmission links, has considerably driven the development of optoelectronic devices based on gallium arsenide technology. A good example of these are found in present InGaAs semiconductor lasers [1], [2].

However, in spite of the high technological standard, totally monochromatic laser sources have not been obtained yet. This aspect is important both in coherent transmission systems (PSK and FSK) and in those where wavelength multiplexation is used.

In this paper we suggest, as an optical source, the use of a structure basically composed of a travelling wave semiconductor optical amplifier with external feedback by means of a monomode optical fibre section. The design also includes two optical devices: an isolator and a beam splitter. Our fundamental aim is to show the feasibility of this design as a high performance laser source.

In the following section the block diagram of the optical source is shown, specifying all the elements included in it. The fundamental equations describing the working principle of the fedback optical amplifier is commented in section 3. In section 4 we present the results obtained from computer simulations. Specifically, curves showing the light-current characteristic and the spectral quality of the emitted optical power have been obtained. Special emphasis is laid on the importance that both TWOA's length and the feedback factor have on the performances of the optical source. From all this, design conditions arise from which optimal behaviours can be found. In section 5 the main conclusions are summarized.

\section{Implementation of travelling wave optical amplifier with ex- ternal feedback}

In Fig.1 the configuration and the elements of the optical source that we are to characterize can be seen. Now we will describe the main function of each of them, leaving for the next section the main equations and some practical aspects.

The optical amplifier (TWOA) is the element capable of contributing the necessary gain to the structure. We have denominated Gs its simple pass gain, which we suppose compensates for all kind of losses not included a priari in the configuration shown in Fig.1 (for example, posible losses of diferent couplings, small losses in the fibre section, etc). In practical situations we use near travelling wave optical amplifiers, although, due to the very low 
reflectivities (lower than $10^{-4}$ ), its influence is irrelevant [3].

The mission of the ideal optical power splitter (BS) would be to break up the power emitted by the amplifier $(\mathrm{Pb})$ in two parts: one delivered in the output of the structure (Pout) and the other one is fedback $\left(P_{c}\right)$. We have denominated ar the fraction of optical power which is fedback and, consequently, (1-ar) the fraction at the output of the source.

The external optical feedback is done by means of a monomode optical fibre section. Due to the wide gain bandwidth and the low attenuation of present fibres, we can suppose that this section neither distorsionates nor attenuates the fedback optical power. However, its length has to be as short as possible and must also fulfil the adecuate phase conditions so that the total increment of the phase of the electrical field along an oscillation will be nil. This condition is set by the lengths and the refraction indexes both the TWOA and of the optical fibre. In that way the resonant modes of the structure and the corresponding spectral distribution of the output optical power will be determined.

The external feedback allows us to include an optical isolator (OI) in order to eliminate the spontaneous emission which escapes through the left face of the amplifier. Thus reducing its saturation level and improving both the noise figure and the spectral quality of the optical source. The state of art of technology supplies us with optical isolators with nil insertion losses and with isolation losses greater than $40 \mathrm{~dB}$.

\section{Basic equations}

Taking into account the configuration of Fig. 1 and the function of each one of the blocks described in the previous section, now we are going to analyse the set of equations that characterize the optical source. A priari we will suppose that the source works in steady state conditions and above the oscillation threshold.

The optical power at the output of the amplifier can be expressed as

$$
P_{b}=G_{\text {. }}
$$

where $\mathrm{Pa}$ is the input power and $\mathrm{Gs}$ the single pass gain of the TWOA. On the other hand, the $\mathrm{BS}$ splits the $\mathrm{Pb}$ power in the following fractions:

$$
\begin{aligned}
& P_{\text {out }}=\left(1-a_{r}\right) P_{b} \\
& P_{c}=a_{r} P_{b}
\end{aligned}
$$

where ar is the fraction of fedback optical power as we have already indicated in section 3. We consider that both the optical fibre sectio and the oI don't present any losses (if this is not the case in a practical situation, we could demand an additional small increment to the $G$ value in our model). Then

$$
P_{b}=P_{c}
$$

By means of the equation set $(1-4)$, it is easy to establish the next fundamental relations:

$$
\begin{gathered}
G_{s}=\frac{1}{a r} \\
P_{\text {out }}=\left(1-\frac{1}{G_{s}}\right) P_{b}
\end{gathered}
$$

Equation (5) implies that the single pass gain of the amplifier is determined by the chosen value of ar. Actually, this equation is the amplitude condition that any oscillator has to fulfil (the value ar finds its corresponding one in the facet reflectivities of a simetric FP cavity laser). The numerical simulations that we have done demostrate that, in order to obtain best performances of the optical source, the value of ar has to lie between 0.3 and 0.4. Consequently, the amplifier single pass gain has values lying between 2.5 and 3.3. If we have already determined the value of $G_{3}$, equation (6) implies that the output power Pout is determined by the $\mathrm{Pb}$ power. This magnitude depends on the bias current (I) and on a set of physical and geometrical parameters. Then, it is necessary to know the internal amplification mechanism of the light. Due to the saturation process that the amplifier experiences (nonlinear behaviour) the co- 
rresponding analysis of the two fundamental equations that took part (the rate equations for the photons and carrier densities) is extremely complicated. They are:

$\frac{\mathrm{dS}\left(z, \lambda_{1}\right)}{\mathrm{dz}}=\frac{\Gamma \beta\left(\lambda_{1}\right)}{2 v}-\frac{\mathrm{N}(z)}{\mathrm{v} \tau_{\mathrm{sp}}}+g\left(z, \lambda_{1}\right) S\left(z, \lambda_{1}\right)$

$\frac{d N(z)}{d z}=\frac{I}{v q d W L_{0 A}}-\frac{N(z)}{v \tau_{s p}}-\sum_{i} g_{m}\left(z, \lambda_{1}\right) S\left(z, \lambda_{1}\right)$

In these equations $S\left(z, \lambda_{1}\right)$ represents, for the $\mathrm{i}$-mode with wavelength $\lambda_{1}$, the photon density $\left(\mathrm{cm}^{-3}\right)$ in the $z$-coordenate (amplification axis). $\mathrm{N}(\mathrm{z})$ is the carrier density $\left(\mathrm{cm}^{-3}\right)$, which decreases from $z=0$ to $z=L O A$. The magnitude $\mathrm{V} / \mathrm{W} \cdot \mathrm{LOA})$ represents the uniform injected current density $\left(\mathrm{kA} / \mathrm{cm}^{2}\right)$ along the active layer, which has a width $W$ and a length LOA (an ideal efficiency of the pumping current $I$ has been supposed ). The constants $\Gamma, \beta, \tau$ sp and $v$ are typical of the semiconductor material. They are the carrier lifetime and the propagation velocity in the active layer, respectively. We denote $q$ as the nominal electron charge.

On the other hand, the net gain per length unit, $g\left(z, \lambda_{1}\right)$, can be related to the intrinsic gain of the semiconductor material, $g m\left(2, \lambda_{1}\right),[4]$, through

$$
g\left(z, \lambda_{1}\right)=\Gamma \cdot g_{m}\left(z, \lambda_{1}\right)-\alpha
$$

where $\alpha\left(\mathrm{cm}^{-1}\right)$ is the material lineal losses coefficient (scattering and other losses mechanisms). The gain $\mathrm{gm}\left(z, \lambda_{1}\right)$ is

$$
g_{m}\left(z, \lambda_{1}\right)=\sigma \cdot\left(N(z)-N_{0}\right)-\gamma \cdot\left(\lambda_{1}-\lambda_{p}\right)^{2}
$$

where $\sigma, \gamma, \quad$ No and $\lambda_{p}$ are important parameters of the material which represent the differential gain parameter, a parabolic parameter, the transparency carrier density and the peak wavelength, respectively.

In order to solve the nonlinear and coupled set of equations given by (7) and (8), the authors of this paper have used the computer model proposed by D.Marcuse in [4]. It is a very powerful technique because it takes into account both the saturation effect and the spatial variation of the carrier density along the $z$-axis. This model has been applied to the proposed structure taking into account the equation set (1-6). Essencially, these equations correspond to the boundary conditions of the differential equations (7) and (8). For example, the photon densities at $z=0$ and $z=$ LoA are proportional to the powers $\mathrm{Pa}$ and $\mathrm{Pb}$. It is easy to demostrate that the minimun pumping current to reach the oscillation condition of the structure (threshold current), is

$$
I_{t h}=\frac{q W d L_{O A}}{\tau_{s p}}\left(N_{0}+\frac{\alpha+\frac{1}{\operatorname{LOA}} \ln \left(\frac{1}{a_{r}}\right)}{\Gamma \sigma}\right)
$$

In all the simulations done we always suppose values of the current I above Ith.

\section{Performances of the designed optical source}

The model developed in [4] has been fitted to the structure shown in Fig.1 and has been numerically solved using the values that we present in table 1. All of them are the typical values that a TWOA has, excepting $a_{r}=0.32$, the length of the fibre section Lof $=666 \mu \mathrm{m}$ and its refraction index nof $=1.5$ (the refraction index of the semiconductor active layer is denoted nTw).

As we show next, it is important to choose a suitable value of the TWOA's length. It can be seen in Fig.2 in which the output power Pout as a function of the optical amplifier's length is represented, for three different values of the bias current. Obviously, the higher the bias current the greater output power obtained. As in a FP lasers, always exists a length for which maximum power is obtained.

In order to analyse the spectral quality of the emitted light, it is necessary to define a merit figure. Then, we define the spectral quality (SQ) as

$$
S Q(d B)=10 \log \left(P_{0} / P_{1}\right)
$$


Figure 1
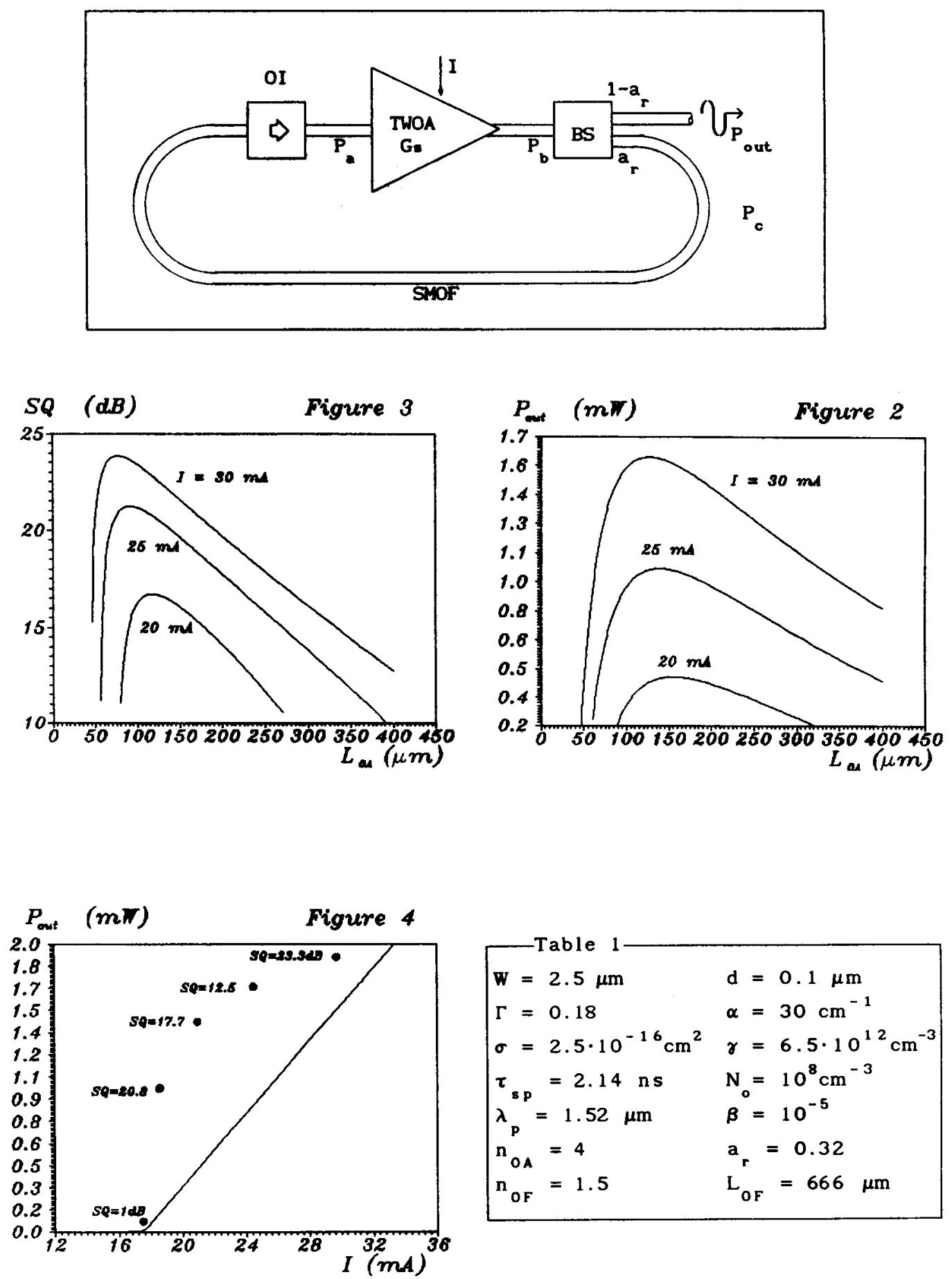

$$
\begin{array}{|ll}
\hline \text { Table } 1 & \\
\Gamma=2.5 \mu \mathrm{m} & \mathrm{d}=0.1 \mu \mathrm{m} \\
\sigma=2.5 \cdot 10^{-16} \mathrm{~cm}^{2} & \gamma=6.5 \cdot 10^{12} \mathrm{~cm}^{-3} \\
\tau_{\mathrm{SP}}=2.14 \mathrm{~ns} & \mathrm{~N}_{0}=10^{8} \mathrm{~cm}^{-3} \\
\lambda_{\mathrm{P}}=1.52 \mu \mathrm{m} & \beta=10^{-5} \\
\mathrm{n}_{\text {OA }}=4 & \mathrm{a}_{\mathrm{r}}=0.32 \\
\mathrm{n}_{\text {OF }}=1.5 & \mathrm{~L}_{\text {OF }}=666 \mu \mathrm{m}
\end{array}
$$


where $P_{0}$ and $P_{1}$ are the optical powers belonging to the central and the first adyacent modes, respectively. In Fig. 3 we have plotted the $S Q$ as a function of the TWOA's length for three different bias currents. Again we find that optimal lengths exist. However, it must be pointed out the fact that, for a given current, the value of LOA that maximizes Pout is not the same one that maximizes SQ. For example, for a $25 \mathrm{~mA}$ current we find a maximum optical power if LoA $140 \mu \mathrm{m}$. Instead, for the same current, the length that maximizes $S Q$ is LoA $\approx 90 \mu \mathrm{m}$. This interesting trade off has to be solved depending on the concrete application. Anyway, we can see that, for lengths arround $100 \mu \mathrm{m}$ and reasonable currents (some ten $\mathrm{mA}^{\prime} \mathrm{s}$ ), output powers over $1.5 \mathrm{~mW}$ can be obtained with a spectral quality greater that 20dB. Obviously, the performances of the optical source improve with the increase of the bias current, although its value will be limitted for the maximum current density $\left(\mathrm{kA} / \mathrm{cm}^{2}\right)$ that the active layer can resist (some ten $\mathrm{kA} / \mathrm{cm}^{2} \mathrm{~s}$ ). Also, it must be pointed out that this type of optical source needs a really low threshold current (Ith $=17.4 \mathrm{~mA}$ for $a_{r}=0.32$ and $L O A=100 \mu \mathrm{m}$ ).

From the obtained results it is interesting to analyse the light-current characteristic and the spectral quality of the optical source for a TWOA's length equal to $100 \mu \mathrm{m}$. In Fig.4 (in continous line) the output optical power as a function of the bias current is represented. We can see that the threshold current is Ith $=17.4 \mathrm{~mA}$ and the curve has a slope of $0.12 \mathrm{~mW} / \mathrm{mA}$. Some values of the SQ have been calculated for different bias currents. For example, for $I=30 \mathrm{~mA}$, values of $S Q=23.5 \mathrm{~dB}$ and Pout= $1.5 \mathrm{~mA}$ have been obtained. This power, due to the high value of SQ, corresponds practically to the lasing mode $\left(\lambda_{p}=1.52 \mu \mathrm{m}\right)$. Then, it can be said the source is monomode.

\section{Conclusions}

The computational method developed by D. Marcuse has been used in this paper to achieve the computer simulations of the optical source shown in fig.1. The obtained results demostrate the high performances that an optical source designed starting from the external feedback of a travelling wave laser amplifier can offer. We have seen that TWOA's lengths and values of the feedback factor that optimize the proposed design exist. To be exact, for Lo $=100 \mu \mathrm{m}, a r=0.32$ and $I=30 \mathrm{~mA}$, we have an optical source that brings, theoretically, an output power of $1.5 \mathrm{~mW}$ with a spectral quality over $23 \mathrm{~dB}$ and a threshold current arround $17 \mathrm{~mA}$. The fact that the lasing mode is 200 times greater than the first lateral mode guarantee the monomode behaviour of the proposed optical source.

\section{References}

[1] J.C.Simon: "Semiconductor Laser Amplifier for Single Mode Optical Fiber $\mathrm{Co}-$ mmunications", J.Opt.Commun., vol. 4, pp. $87-98,1983$

[2] G.Eisenstein, R.M.Jopson:"Measurements of the gain spectrum of near-travelling-wave and Fabry-Perot semiconductor optical amplifiers at $1.55 \mu \mathrm{m} "$,Int. J.Electronics, Vol.60, No.1, pp. 113121,1986

[3] M.J.O'Mahony:"Semiconductor Laser Optical Amplifiers for Use in Future Fiber Systems", IEEE J.Lightwave Tech. Vol.6, No.4, pp.531-544, 1988

[4] D.Marcuse: "Computer Model of an Injection Laser Amplifier", IEEE J.Quantum Elec., Vol.13,No.8, pp.63-73, 1983 